\title{
Changes of Fluctuating Asymmetry with Age in Human Fetuses and Young Infants
}

\author{
Stefan Van Dongen ${ }^{1, *}$, Claartje Ten Broek ${ }^{1,2}$, Jessica Bots ${ }^{1}$ and Frietson Galis ${ }^{2}$ \\ 1 Evolutionary Ecology Group, Campus Drie Eiken, University of Antwerp, Universiteitsplein 1, 2610 Wilrijk, \\ Belgium; claartje.tenbroek@naturalis.nl (C.T.B.); jessica.bots@uantwerpen.be (J.B.) \\ 2 Naturalis Biodiversity Center, Endless Forms, Vondellaan 55, 2332 AA Leiden, The Netherlands; \\ frietson.galis@naturalis.nl \\ * Correspondence: stefan.vandongen@uantwerpen.be; Tel.: +32-03-265-33-36
}

Academic Editor: John H. Graham

Received: 6 February 2017; Accepted: 14 March 2017; Published: 21 March 2017

\begin{abstract}
Background: Developmental instability (DI), often measured by fluctuating asymmetry (FA), increases with stress in humans, yet little is known about how stress affects the changes of asymmetry with age. More specifically, it is unknown if fetuses experiencing a major congenital abnormality will express higher FA already during early development or only at a later age; (2) Methods: We combine two datasets to study associations between age and asymmetry in human fetuses and young infants. One population consists of fetuses from spontaneous abortions and early deceased infants where many experienced major congenital abnormalities, and a second from elicited abortions for social reasons; (3) Results: While the occurrence of major abnormalities did not seem to affect the way asymmetry decreased with age, differences between the two populations were observed; and (4) Conclusions: In one population where fetuses and young infants deceased of natural causes, asymmetry decreased rapidly until 20 weeks of age and then leveled off. Over the entire timespan (week 15-49), individuals with major congenital abnormalities showed higher FA, suggesting that developmental perturbations increase FA rapidly. In the second, more normal population with abortions solicited for social reasons, the decrease in asymmetry with age was less profound and not statistically significant, calling for further research toward understanding regional differences.
\end{abstract}

Keywords: developmental instability (DI); fluctuating asymmetry (FA); human fetuses and infants; age; congenital abnormalities

\section{Introduction}

Developmental instability (DI), the inability of an organism to buffer its development against developmental noise [1], is often measured through random deviations from perfect symmetry (the so called fluctuating asymmetry (FA) [2]). FA has often been studied for its associations with various estimates of stress, fitness and health, and this in a wide variety of organisms, including plants, invertebrates, and vertebrates including humans [3]. Overall, it has often been argued that the strengths of associations vary a lot among studies and that it seems impossible to predict if and when FA will show associations with measures of stress, fitness or health. Indeed, in a meta-analysis of the human literature, on average a robust effect was detected, but that variation among studies remained largely unexplained [3]. Associations between FA and stress were strongest in human fetal studies, although that levels of stress may need to be very high [3-5].

While associations between FA and various types of stress, health and fitness have been studied intensively, surprisingly relative little is known about the effects of stress on the changes of FA with age. Indeed, longitudinal studies are very rare, but also cross-sectional studies have not often focused 
on the associations of FA with age (see, e.g., [6-8]). Also in humans, the literature on the changes of FA with age are scarce, especially in fetuses. Nevertheless, such insights can provide valuable information with respect to the mechanisms causing increases of FA with stress. More specifically, the morphogenetic drift model for skeletal asymmetry predicts that effects of stress on FA will accumulate over time, such that associations between stress and FA will emerge later in life [9]. Under this model, one thus can predict that a higher FA in fetuses experiencing congenital abnormalities serious that affect normal functioning and are potentially lethal, i.e., major congenital abnormalities (as observed earlier, [10]), will become apparent only at the later developmental stages. One can thus predict an interaction between age and stress. In this study, we combine data from two populations, one with fetuses from spontaneous abortions and still births and the other from elective abortions. While the first population contained many fetuses with major abnormalities, the latter did not. A detailed analysis of the associations between FA and age is presented and comparisons between populations, and between fetuses who did or did not experience developmental perturbations leading to major abnormalities.

\section{Materials and Methods}

We combine data from two samples in thus study, of which all traits measured in both populations were analyzed here. The first sample was collected in Turku (Finland), the second in Amsterdam (The Netherlands).

Between 1964 and 1974, human fetuses from elective abortions were collected by scientists at the Institute of Dentistry, University of Turku (Finland) to study craniofacial development (further called Turku). For ethical reasons all background information on the mothers had been discarded, leaving data on the age, sex and size for the majority of the fetuses. Patient data and radiographs were used according to the guidelines of the Medical Ethics Committee of the VU University Medical Center and patient anonymity was strictly maintained. Parental written informed consent was obtained for patients and data were handled in a coded and completely anonymous fashion, according to Dutch national ethical guidelines (Code for Proper Secondary Use of Human Data, Dutch Federation of Medical Scientific Societies; http://www.federa.org/codes-conduct). Ethical approval (approval number 648/32/300/05) to study the human collection of embryos and fetuses owned by the University of Turku was obtained from the National Supervisory Authority for Welfare and Health of Finland (VALVIRA).

Complete fetuses were preserved by the 'clearing and single staining' procedure and kept on glycerin. This technique allows us to study skeletal traits by making eviscerated specimens semi-transparent with potassium hydroxide and staining ossified parts with alizarin red. All fetuses were externally evaluated for skeletal malformations (including craniofacial and limb defects) by a pediatric pathologist and no major abnormalities were reported. Major congenital abnormalities (i.e., compromising the normal functioning and sometimes potentially lethal), indicate major disturbances of development starting during the very early organogenesis and include for example the absence of one or both kidneys, skeletal dysplasias, extreme scoliosis, anencephaly, cleft lip/palate, cyclops, dextroposition of the heart, monoventricular or monoarterial heart; atresia of the aorta; chromosomal abnormalities, amnion disruption syndrome, chondrodysplasia and sirenomalia [10]. In many cases, several (major) congenital abnormalities co-occur, and in a few exceptional fetuses it is not always possibly to identify abnormalities very precisely. It is important to note that the presence of these major abnormalities cannot be missed during medical examinations. As such, we are certain that in this first population did not experience major congenital abnormalities, in contrast to population 2 (see below). A high frequency of cervical ribs and rudimentary 12th ribs was reported [11]. Measurements were collected from 178 individuals, 95 females and 83 males, aged 10-21 weeks (average 15.3 weeks \pm 2.1 weeks). Each fetus' left and right hand, arm, leg and back were photographed twice independently and repeatedly with a Canon 300D digital SLR camera (Canon, Machelen, Belgium). Digital pictures were scaled using a transparent ruler (resolution of $0.1 \mathrm{~cm}$, used for scaling purposes) and analyzed in Image J 1.42q (National Institutes of Health, Bethesda, MA, USA). 
Figure 1 provides an overview of the measured traits, which include the size of the humerus, radius, ulna, metacarpal 2, 3, 4 and -5, femur, tibia, fibula, and scapula. For each picture, first, two landmarks were placed on the ruler at a distance of $2 \mathrm{~cm}$ to allow scaling measurements on different pictures to the same unit (i.e., in centimeters). Next, landmarks were placed at the center of the proximal and distal ends of the bones, allowing us to calculate the length of each bone on the basis of these two coordinates (i.e., as a Euclidian distance). All landmarks were placed twice independently for each picture to evaluate repeatability. Variation in bone lengths between measurements of repeated pictures of the same bone will allow us to evaluate the degree of measurement error (ME) due to repositioning of the fetus and taking pictures. In addition, variation in bone lengths between repeated measurements within pictures will allow us to evaluate the degree of measurement error due to placing landmarks (see statistical analyses).
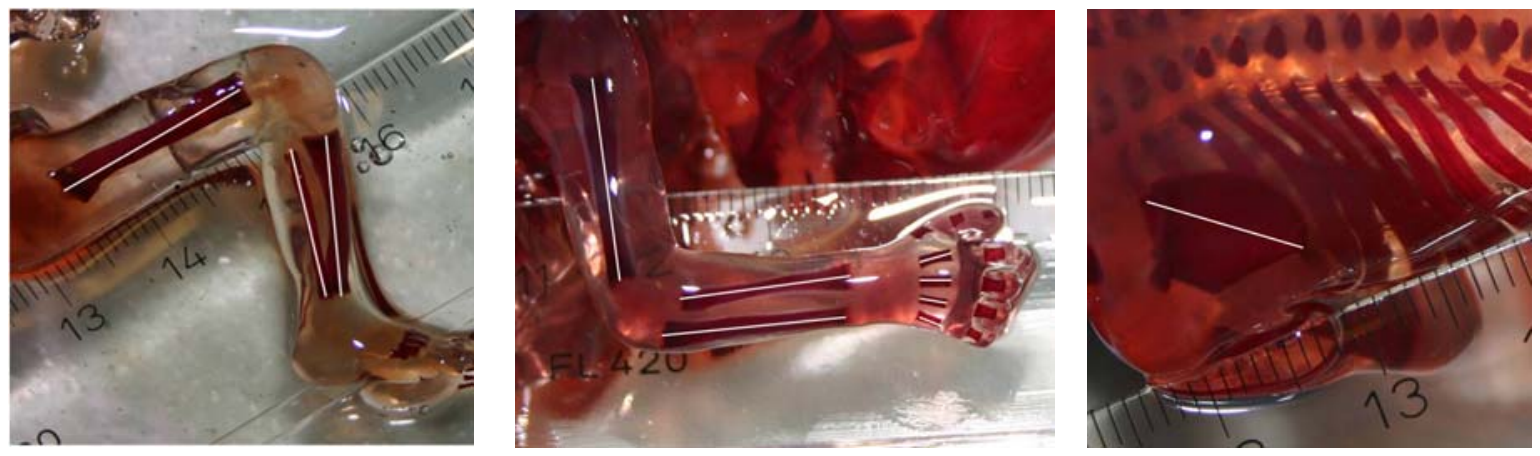

Figure 1. Photographs of leg (left picture), arm and hand (middle) and scapula (right) of alizarin colored fetuses. White lines indicate measurements of limb bones and metacarpals from the midpoint of the proximal end of the bones to the midpoint of the distal end. The scapula was measured from the inferior to the medial angle.

Since 1980, all deceased infants and fetuses have been routinely radiographed both ventrally and laterally, when presented for autopsy at the VU Medical Centre (23 mA, 70-90 kV, 4-12 s, Agfa (Mortsel, Belgium) Gevaert D7DW Structurix films) (further called Amsterdam). This research was carried out on the anterior-posterior projections of 1339 deceased fetuses and infants obtained between 1990 and 2009. In total, we measured 616 male and 487 female fetuses and infants and 4 fetuses of unknown sex (13-49 weeks, mean: 26.1 weeks \pm 8.0 weeks). We excluded some fetuses when radiographs had insufficient resolution or limb bones were not properly positioned. The radiographs were digitized using a Canon 30D digital camera in a fixed-distance set-up with a glass plate and a flash underneath. We measured the length of the left and right digits 2 and 4, metacarpals 2, 3, 4 and 5, humerus, femur, fibula, radius, ulna and tibia from the midpoint of the proximal end of the bone to the midpoint of the distal end of the bone in Image J version 1.42q. Digits were measured from the proximal end of the proximal phalanx to the distal end of the distal phalanx. All measurements were obtained with a transparent ruler up to $0.01 \mathrm{~cm}$ accurate. We excluded all fetuses that had abnormally developed limbs from analyses, because of the likely direct effect of development on the asymmetry of the limbs. Standard autopsy reports were made by pathologists and filed in a national pathological archive (PALGA), on the basis of which major abnormalities were identified (see above and [10]). Figure 2 provides an overview of the measurements obtained.

Some traits were not included in our final analyses. Scapula of the Turku population, and digits 2 and 4 of the Amsterdam population were excluded because they were not measured in both populations. In addition, in order to avoid that the metacarpals would be overrepresented in the calculation of the average FA across traits, metacarpals 3 and 5 were omitted from both populations. Of all remaining traits, a mixed regression model analysis [12] was performed with side as continuous covariate (left $=-0.5$ and right $=0.5$, such that a positive slope would indicate that the right side 
was lower than the left), and both individual and photograph nested within individual as random effects. Two-way interactions between these random effects and side were also included and their respective variances reflect true FA and measurement error due to taking photographs and positioning the fetus respectively. The residual variance reflects measurement error due to the measurements within single photographs. Unbiased individual and trait specific signed FA values corrected for measurement error and directional asymmetry (DA, as estimated by the fixed side effect) were obtained as the best linear unbiased prediction (BLUP) of the random slopes from the individual $\times$ side random effects interaction. These signed FA values showed normal to leptokurtic distributions, showing that there was no antisymmetry. Unsigned FA values, obtained as the absolute value of the signed FA, were divided by trait size because the uncorrected unsigned FA correlated positively with size $[4,5]$. Average unbiased and size corrected FA was obtained as the average of the standardized size corrected unsigned FA's [10,12]. Associations between average FA and age was studied using two approaches. First, a polynomial regression was performed up to the fifth degree. In addition, population and whether or not a fetus experiences a major abnormality were added as factors as well as their interactions with age and the polynomial age terms. The model was simplified using a stepwise backward elimination. Second, a generalized additive model was fitted separately for both populations. For the Amsterdam population, the analysis was done separately for fetuses with or without major abnormalities. The added value of this second approach is that it does not assume any parametrical form of the association between average FA and age. Finally, we also analyzed the FA-age associations for fetuses aged 21 weeks or younger, thus excluding the older fetuses in the Amsterdam population. We also focus on the changes in FA between weeks 10 and 21, a period for which we have data for both populations and with the strongest change in FA (see below). All analyses were performed in the package R (R Core Team, Vienna, Austria) [13].
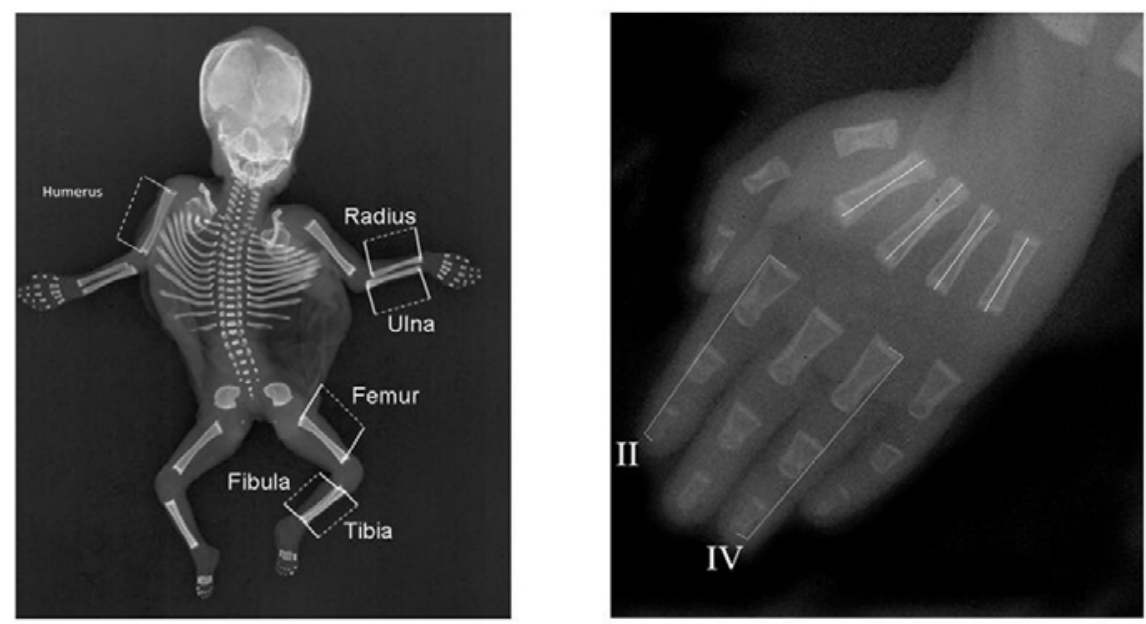

Figure 2. Left: Radiograph of an entire fetus. Lines indicate measurements of limb bones from the midpoint of the proximal end of the bones to the midpoint of the distal end; Right: Radiograph of right hand. Lines indicate length of the midpoint of the proximal end of the proximal phalanx to the midpoint of the distal end of the distal phalanx for digits 2 and 4. Metacarpals 2, 3, 4 and 5 were measured from the midpoint of the proximal end of the bones to the midpoint of the distal end.

\section{Results}

\subsection{Fluctuating Asymmetry, Measurement Error and Directional Asymmetry}

Table 1 provides an overview of the results from the mixed regression analyses. Overall we can conclude that measurement error was relatively small except for the metacarpals in the Amsterdam population. ME was generally smaller in the Turku population. There was a consistent degree of statistically significant directional asymmetry in 5 bones of the arms and legs (but not in femur and 
metacarpals) in the Turku population, where the right hand side was slightly longer. This was not seen for the Amsterdam population.

Table 1. Overview of the degree of measurement error (ME) and directional asymmetry (DA). Variance components of the mixed regression models (see text for details) reflecting true fluctuating asymmetry (FA), measurement error due to taking photographs and positioning of the fetuses (ME-photo) and measurement error due to the actual measurements of the bone lengths (ME-measurement) are provided. In addition, the slope of the mixed regression model, reflecting the degree of DA and its standard error are also given. Significant DA $(p<0.05)$ are indicated in bold.

\begin{tabular}{ccccccccc}
\hline Population & \multicolumn{1}{c}{ Amsterdam } & \multicolumn{5}{c}{ Turku } \\
\hline Trait & FA & ME-Photo & $\begin{array}{c}\text { ME- } \\
\text { Measurement }\end{array}$ & DA & FA & ME-Photo & $\begin{array}{c}\text { ME- } \\
\text { Measurement }\end{array}$ & DA \\
\hline Radius & 1.07 & 0.07 & 0.56 & $\begin{array}{c}-0.0025 \\
(0.0014)\end{array}$ & 1.00 & 0.10 & 0.02 & $\begin{array}{c}-\mathbf{0 . 0 1 9} \\
\mathbf{( 0 . 0 0 2 5 )}\end{array}$ \\
\hline Ulna & 1.4 & 0.71 & 0.23 & $\begin{array}{c}-0.0032 \\
(0.0016)\end{array}$ & 1.2 & 0.09 & 0.06 & $\begin{array}{c}-\mathbf{0 . 0 1 8} \\
\mathbf{( 0 . 0 0 2 7 )}\end{array}$ \\
\hline Humerus & 19.6 & 5.92 & 0.36 & $\begin{array}{c}\mathbf{0 . 0 1 5 8} \\
\mathbf{( 0 . 0 0 6 2 )}\end{array}$ & 1.90 & 0.75 & 0.29 & $\begin{array}{c}\mathbf{- 0 . 0 1 0 9} \\
\mathbf{( 0 . 0 0 3 8 )}\end{array}$ \\
\hline Tibia & 5.40 & 0.07 & 0.13 & $\begin{array}{c}-0.0008 \\
(0.0023)\end{array}$ & 0.81 & 0.39 & 0.04 & $\begin{array}{c}-\mathbf{0 . 0 0 5 3} \\
\mathbf{( 0 . 0 0 2 5 )}\end{array}$ \\
\hline Fibula & 2.77 & 0.93 & 0.23 & $\begin{array}{c}-0.0047 \\
(0.0027)\end{array}$ & 0.48 & 0.07 & 0.08 & $\begin{array}{c}-\mathbf{0 . 0 0 6 0} \\
\mathbf{( 0 . 0 0 1 9 )}\end{array}$ \\
\hline Femur & 2.2 & 0.68 & 0.31 & $\begin{array}{c}4.58 \\
\mathbf{( 0 . 0 6 9 )}\end{array}$ & 2.34 & 0.26 & 0.19 & $\begin{array}{c}0.002 \\
(0.004)\end{array}$ \\
\hline meta2 & 0.53 & 0.15 & 0.51 & $\begin{array}{c}-0.0008 \\
(0.0016)\end{array}$ & 0.15 & 0.02 & 0.01 & $\begin{array}{c}-0.001 \\
(0.001)\end{array}$ \\
\hline meta4 & 0.36 & 0.17 & 0.20 & $\begin{array}{c}0.0006 \\
(0.0012)\end{array}$ & 0.19 & 0.03 & 0.01 & $\begin{array}{c}0.0010 \\
(0.0012)\end{array}$ \\
\hline
\end{tabular}

\subsection{Associations between Fluctuating Asymmetry and Age}

Average size corrected unbiased FA's decreased with age in both populations and could be modelled with a quadratic model. The degree of decrease differed significantly as indicated by the significant interactions between age and age squared with population (Table 2). After plotting the associations on the basis of the parameter estimates of the polynomial regression model (Table 2), we can conclude that the decrease in average FA was steeper in the Amsterdam population (Figure 3). This comparison could only be made for fetuses aged between 10 and 21 weeks old because the Turku population did not contain any data from older fetuses. For the Amsterdam population, the decrease leveled off after about 23 weeks and remained largely unchanged (Figure 3). Fetuses with major abnormalities showed consistently larger average FA, but the change in FA over time did not show significant differences between fetuses with or without major abnormalities (i.e., no significant interactions, Table 2). A very comparable pattern was observed when analyzing the associations between average FA and age separately for the two populations and for fetuses and infants with or without major abnormalities in the Amsterdam population using a generalized additive model (Figure 3). When analyzing only fetuses of comparable ages (between 10 and 21 weeks) from both populations, the age-population interaction was confirmed $\left(t_{412}=2.87, p=0.004\right)$. Average FA decreased significantly in the Amsterdam dataset (slope $=-0.11$, standard error $(\mathrm{SE})=0.02, \mathrm{t}_{295}=-6.35$, $p<0.0001$ ) but not in the Turku population (slope $=-0.03, \mathrm{SE}=0.02, t_{295}=-1.38, p=0.19$ ) (Figure 4). The quadratic or higher order effects of age did not contribute significantly in explaining variation in FA (all $p$-values > 0.2). Thus, changes in FA within this range of ages (10-21 weeks) appeared linear. Fetuses with major abnormalities had on average a higher level of FA, but not significantly so in this smaller dataset $\left(t_{412}=1.38, p=0.16\right)$. The magnitude of the difference was almost identical to that from the analyses of the complete dataset (difference $=0.10, \mathrm{SE}=0.08$, Table 2 ). 
Table 2. Overview of the model building and parameter estimates of the polynomial regression of the association between average FA and age, and to what extend it differs between populations (Turku vs. Amsterdam) and between fetus with or without major abnormalities. Statistically significant terms are highlighted in bold.

\begin{tabular}{|c|c|c|c|c|}
\hline Model Parameter & Estimate (SE) & $t$-Value & d.f. & $p$-Value \\
\hline Intercept & $10.1(1.83)$ & 5.50 & 1083 & $<0.0001$ \\
\hline Age & $-1.33(0.27)$ & -5.02 & 1083 & $<0.0001$ \\
\hline Population & $-5.31(1.91)$ & -2.78 & 1083 & 0.006 \\
\hline major abnormality & $0.10(0.03)$ & 3.19 & 1085 & 0.001 \\
\hline age $^{2}$ & $0.068(0.014)$ & 4.60 & 1085 & $<0.0001$ \\
\hline age $^{3}$ & $-0.0015(-0.0003)$ & -4.31 & 1085 & $<0.0001$ \\
\hline age $^{4}$ & $0.00001(0.000003)$ & 4.09 & 1085 & $<0.0001$ \\
\hline $\operatorname{age}^{5}$ & - & -0.37 & 1084 & 0.765 \\
\hline age: population & $0.56(0.23)$ & 2.38 & 1085 & 0.017 \\
\hline age $^{2}$ : population & $-0.014(0.007)$ & -2.45 & 1085 & 0.015 \\
\hline age $^{3}$ : population & - & 0.883 & 1083 & 0.377 \\
\hline age $^{4}$ : population & - & 0.395 & 1082 & 0.693 \\
\hline age $^{5}$ : population & - & -0.05 & 1081 & 0.956 \\
\hline age: major abnormality & - & -0.33 & 1080 & 0.745 \\
\hline age $^{2}$ : major abnormality & - & -0.09 & 1079 & 0.978 \\
\hline age $^{3}$ : major abnormality & - & -1.08 & 1078 & 0.280 \\
\hline age $^{4}$ : major abnormality & - & 1.63 & 1077 & 0.110 \\
\hline age $^{5}$ : major abnormality & - & -1.53 & 1076 & 0.121 \\
\hline
\end{tabular}

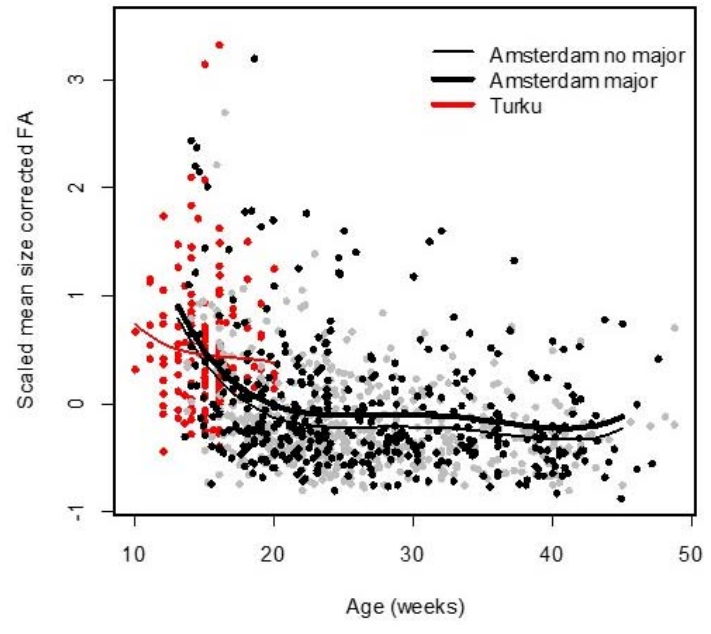

(a) polynomial regression results

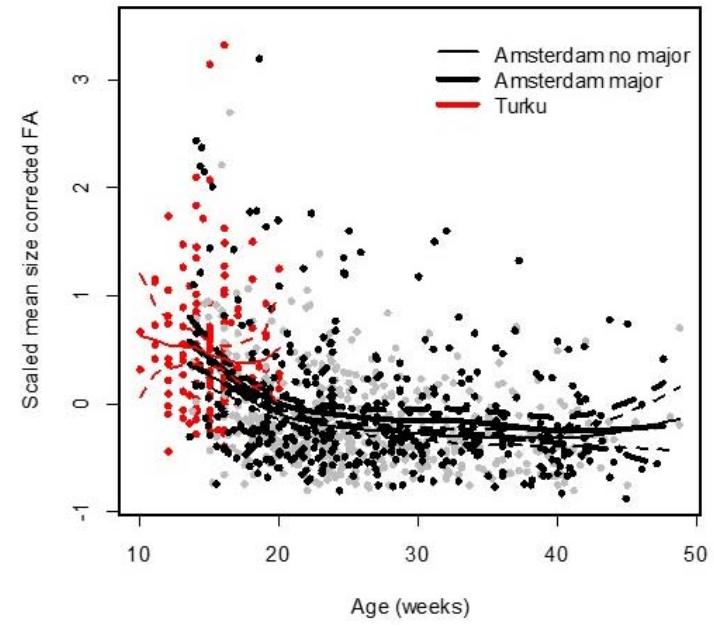

(b) generalized additive model results

Figure 3. Associations between average FA and age in two populations (Amsterdam and Turku (red symbols and lines). For the Amsterdam population, separate curves are provided for fetuses with (black symbols thick black lines) or without (grey symbols and narrow black lines) major abnormalities. The left plot provides the results based on a polynomial regression model (Table 2). The right plot shows the results from a generalized additive model and includes confidence bands. 


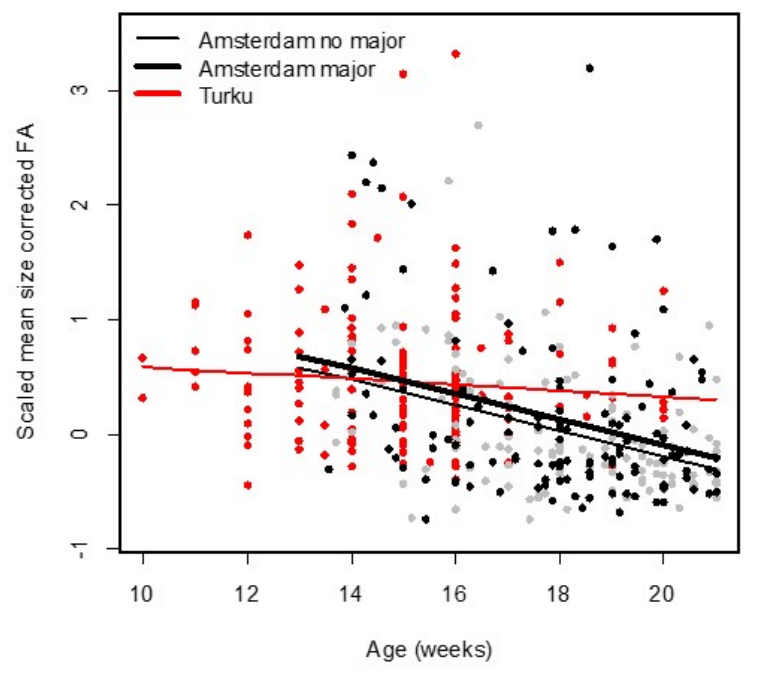

Figure 4. Associations between average FA and age in two populations (Amsterdam and Turku) for fetuses of 21 weeks or younger.

\section{Discussion}

We studied associations between DI, as measured by FA in limbs and hands, and age in human fetuses and infants. FA was on average larger in the Turku population, but this was to some extent due to the age differences between the two populations. Nevertheless, we observed a different change in FA between the youngest (age $<21$ weeks) fetuses of both populations, where the decrease was less steep, and not statistically significant for the Turku population. This result is somewhat unexpected given that the fetuses from Turku were obtained from elected abortions and thus reflect fetuses following a relatively normal development compared to those from spontaneous abortions in the Amsterdam population. About the reasons for this difference we can only speculate because both populations may differ in several other aspects. One possible explanation could be our inability to measure very young fetuses in the Amsterdam population, because the bones were not sufficiently ossified to get X-rays of sufficiently high quality at the very young ages. The youngest fetuses in the Turku were 10 weeks old (and the oldest 21 weeks) while in the Amsterdam population, ages ranged between 13 and 49 weeks, and about $70 \%$ of them were older than 21 weeks. As the fetuses that die at this very young age (i.e., before week 10 or with insufficient ossification between week 10 and 20) are (partly) missing from our Amsterdam sample, yet, may also reflect those who experience the most severe congenital abnormalities. This may have biased our estimate of FA in the Amsterdam sample downward to some extent. In addition, we cannot exclude differences between the two methods of measuring (X-ray vs. stained fetuses) in spite of the fact that the FA values were corrected for the differences in measurement error. More specifically, shrinkage of tissue due to staining in the Turku population may have biased our FA estimate upward in this population. More research with data from different populations might shed more insights in this potential between-population difference. On the other hand, it is also worth noting that the use of FA as a measure of DI has been questioned and may not be correct generally, as outlines in a recent review [14]. Nevertheless, we did find higher FA in fetuses and infants with major abnormalities ([10] and this study), with severe genetic abnormalities and when several organ systems or patterning processes are affected [5], which does suggest that limb FA does signal developmental perturbations, albeit often only when they are very severe.

In the Amsterdam population, the decrease in FA with age appeared linear for the younger ages (13-21 weeks) and then leveled off around the age of 23 weeks. While fetuses and infants with major abnormalities showed higher FA, as seen previously in a smaller sample from this population [9], the associations of FA with age were parallel for fetuses and infants with or without major abnormalities. We found no evidence that FA-age associations are affected by major abnormalities which typically 
emerge during early development, and thus could not demonstrate a significant statistical interaction between age and the occurrence of major abnormalities as predicted by the morphogenetic drift model for skeletal asymmetry [8]. To what extent the differences in FA between fetuses who did and did not have major congenital abnormalities, would increase later in life, could not be studied here since all fetuses died prematurely or as very young infant. More research should focus on the comparison of changes in FA through development for groups of individuals differing in the amount of developmental perturbations.

Acknowledgments: We thank Jaap van Veldhuisen and Ron Otsen of the photography division of the Institute of Pathology of the VU University Medical Centre (VUMC) in Amsterdam, the Netherlands.

Author Contributions: Stefan Van Dongen, Claartje tenBroek., Jessica Bots and Frietson Galis conceived and designed the experiments; Claartje tenBroek, J.B. and F.G. performed the measurements; Stefan Van Dongen analyzed the data and Stefan Van Dongen, Claartje tenBroek, Jessica Bots and Frietson Galis wrote the paper.

Conflicts of Interest: The authors declare no conflict of interest.

\section{References}

1. Klingenberg, C.P. A developmental perspective on developmental instability: Theory, models, and mechanisms. In Developmental Instability: Causes and Consequences; Polak, M., Ed.; Oxford University Press: Oxford, UK, 2003; pp. 14-34.

2. Ludwig, W. Rechts-Links-Problem im Tierreich und beim Menschen, mit Einem Anhang; Springer: Berlin, Germany, 1932. (In German)

3. Van Dongen, S.; Gangestad, S.W. Human fluctuating asymmetry in relation to health and quality: A meta-analysis. Evol. Hum. Behav. 2011, 32, 380-398. [CrossRef]

4. Bots, J.; ten Broek, C.M.A.; Belien, J.A.M.; Bugiani, M.; Galis, F.; Van Dongen, S. Higher limb asymmetry in deceased human fetuses and infants with aneuploidy. Sci. Rep. 2014, 4. [CrossRef] [PubMed]

5. Ten Broek, C.M.A.; Bots, J.; Bugiani, M.; Galis, F.; Van Dongen, S. Developmental origins of limb developmental instability in human fetuses: Many abnormalities make the difference. Symmetry. Submitted.

6. Hallgrímsson, B. Ontogenetic patterning of skeletal fluctuating asymmetry in rhesus macaques and humans: Evolutionary and developmental implications. Int. J. Primatol. 1999, 20, 121-151. [CrossRef]

7. Møller, A.P.; Van Dongen, S. Ontogeny of asymmetry and compensational growth in elm Ulmus glabra leaves under different environmental conditions. J. Int. Plant Sci. 2003, 164, 519-526. [CrossRef]

8. Palestis, B.G.; trivers, R. A longitudinal study of changes in fluctuating asymmetry with age in Jamaican youth. Symmetry 2016, 8. [CrossRef]

9. Hallgrímsson, B. Fluctuating asymmetry in the mammalian skeleton-Evolutionary and developmental implications. Evol. Biol. 1998, 30, 187-251.

10. Van Dongen, S.; Wijnaendts, L.C.D.; Ten Broek, C.M.A.; Galis, F. Fluctuating asymmetry does not consistently reflect severe developmental disorders in human foetuses. Evolution 2009, 63, 1832-1844. [CrossRef] [PubMed]

11. Bots, J.; Wijnaendts, L.C. D.; Delen, S.; Van Dongen, S.; Heikinheimo, K.; Galis, F. Analysis of cervical ribs in a series of human fetuses. J. Anat. 2011, 219, 403-409. [CrossRef] [PubMed]

12. Van Dongen, S.; Molenberghs, G.; Matthysen, E. The statistical analysis of fluctuating asymmetry: REML estimation of a mixed regression model. J. Evol. Biol. 1999, 12, 94-102. [CrossRef]

13. The R Project for Statistical Computing. Available online: http://www.r-project.org (accessed on 17 August 2016).

14. Klingenberg, C.P. Analyzing Fluctuating Asymmetry with Geometric Morphometrics: Concepts, Methods, and Applications. Symmetry 2015, 7, 843-934. [CrossRef] 